\title{
IS HYPERBILIRUBINAEMIA A CLINICAL USEFUL INDICATOR OF REDUCED DRUG CLEARANCE CAPACITY IN NEONATES?
}

\author{
A. Smits ${ }^{1}$, R. de Cock $^{2}$, B.J. Anderson ${ }^{3}$, G. Palmer ${ }^{4}$, C. Knibbe ${ }^{2}$, K. Allegaert ${ }^{1}$
}

${ }^{\text {I} P a e d i a t r i c s, ~ U n i v e r s i t y ~ H o s p i t a l s ~ L e u v e n, ~ L e u v e n, ~ B e l g i u m, ~}{ }^{2}$ Division of Pharmacology, Leiden University, Leiden, The Netherlands, ${ }^{3}$ Department of Anaesthesiology, University of Auckland, Auckland, New Zealand, ${ }^{4}$ Department of Paediatric Anaesthesia and Pain Management, Royal Children's Hospital, Melbourne, NSW, Australia

Background: There is extensive variability in clearance between neonates, mainly explained by age or size. We aimed to assess if hyperbilirubinaemia also contributes as covariate, using reported paracetamol [1] and propofol datasets [2] since both compounds undergo glucuronidation.

Methods: Population pharmacokinetic analysis of 943 paracetamol observations in 158 neonates was undertaken (NONMEM, two-compartment linear disposition). A similar approach (three-compartment) was performed on a dataset of 235 propofol concentration-time points in 25 neonates. Covariates were postmenstrual and postnatal age (PMA, PNA), weight and indirect hyperbilirubinaemia (dichotomous, PNA adapted fixed cut-off values [3])

Results: For the paracetamol clearance, covariate information predicted $60.9 \%$ of variance [weight 57.5 , PMA 2.2, unconjugated bilirubin $1.2 \%$ ]. For propofol, the age (PMA+PNA) model described the data most accurately. The covariates PMA+PNA explained $67 \%$ of the interindividual variability compared to $45 \%$ with PMA+bilirubin. Introduction of bilirubin into the PMA+PNA model did not improved this model.

Conclusions: Ontogeny itself, reflected by age (PMA, PNA) or size are the dominant covariates of drug clearance in neonates. Hyperbilirubinaemia appears to be only a very modest clinical indicator of (reduced) drug clearance in neonates for drugs that undergo glucuronidation.

[1] Allegaert K et al. Arch Dis Child doi:10.1136/adc.2010.204552;

[2] Allegaert K et al. Br J Anaesth 2007;99:864-70;

[3] Palmer GM et al. Br J Anaesth 2008;101:523-30 